\title{
Riqueza y características morfométricas de aves de sotobosque en Varillal Alto Seco de la Reserva Nacional Allpahuayo Mishana, Perú
}

\author{
Richness and morphometrics characteristics of birds of understory forest in \\ Varillal Alto Seco of the Allpahuayo Mishana National Reserve, Peru \\ Arturo Acosta Diaz ${ }^{1}$, Javier Ayapi-Da-Silva ${ }^{1}$, Marley Ocampo-Rodríguez ${ }^{1}$, \\ Hugo Gálvez Carrilloº,
}

\section{Resumen}

Se evaluaron las aves de sotobosque en el Varillal Alto Seco de la Reserva Nacional Allpahuayo Mishana (RNAM) entre enero y mayo de 2016 para determinar su riqueza y características morfométricas mediante conteo por puntos aplicando observación directa, reconocimiento auditivo y captura con redes de neblina. Se registró una riqueza de 12 órdenes, 18 familias y 122 especies, donde el orden de Passeriformes registró 13 familias y Thamnophilidae con 23 especies. La riqueza de especies entre varillales varió entre 85 especies (varillal B) y 51 especies (varillal C). Los índices no paramétricos indican que la riqueza específica de aves observadas (122 especies) siempre estuvo por debajo de lo esperado (CHao 1 con 130 especies; CHao 2 con 133 especies; Bootstrap con 136 especies y Jackknife 1 con 149 especies) y la misma tendencia se observó con la curva de Clench con 166 especies esperadas, mientras que los índices de diversidad de Simpson variaron entre 0.96 y 0.98 , Shannon-Winner entre 3.69 y 4.30 y Margalef entre 10.31 y 16.93, indicando una alta diversidad de aves. Los datos morfométricos de las especies capturadas muestran baja variabilidad con respecto a lo reportado para otros lugares, excepto Geootrygon montana, Celeus elegans, Pithys albifrons, Schistocicla leucostigma, Xiphorhynchus elegans, Attila spadiceus y Manacus manacus que presentan una mayor variabilidad. Se concluye que el sotobosque del Varillal Alto tiene una riqueza de aves alta en la RNAM.

Palabras clave: varillal alto seco; aves de sotobosque; medidas morfométricas; Reserva Mishana-Allpahuayo

\footnotetext{
${ }^{1}$ Facultad de Ciencias Biológicas, Universidad Nacional de la Amazonía Peruana, Iquitos, Perú

${ }^{2}$ Estación Experimental Iquitos, Centro de Investigación IVITA, Universidad Nacional Mayor de San Marcos, Iquitos, Perú

${ }^{3}$ E-mail:hgalvezc@unmsm.edu.pe
}

Recibido: 29 de julio de 2018

Aceptado para publicación: 24 de enero de 2019 
The understory birds in the Alto Seco Varillal of the Allpahuayo-Mishana National Reserve (RNAM) in Peru were evaluated between January and May 2016 to determine their richness and morphometric characteristics applying direct observation, auditory recognition and capture using mist nets. The richness was composed of 12 orders, 18 families and 122 species, where the order of Passeriformes recorded 13 families and Thamnophilidae 23 species. Species richness among «varillales» varied between 85 species (varillal $\mathrm{B}$ ) and 51 species (varillal $\mathrm{C}$ ). The nonparametric indices indicate that the specific richness of birds observed (122 species) was always lower than expected (CHao 1 with 130 species, CHao 2 with 133 species, Bootstrap with 136 species and Jackknife 1 with 149 species) and the same trend was observed with the Clench curve with 166 expected species, while the Simpson diversity index varied between 0.96 and 0.98, Shannon-Winner between 3.69 and 4.30 and Margalef between 10.31 and 16.93, indicating a high diversity of birds. The morphometric data of the species captured showed low variability with respect to that reported for other places, except Geootrygon montana, Celeus elegans, Pithys albifrons, Schistocicla leucostigma, Xiphorhynchus elegans, Attila spadiceus and Manacus manacus that presented greater variability. It is concluded that the understory of the Alto Seco Varillal has high richness of birds in the RNAM.

Key words: dry high varillal; understory forest birds; morphometric measurements; Allpahuayo-Mishana

\section{INTRODUCCIÓN}

La Reserva Nacional Allpahuayo Mishana (RNAM) alberga y protege una gran riqueza de ecosistemas, caracterizándose por la formación y variedad de bosques sobre arena blanca denominados varillales, los cuales albergan especies que se han adaptado en este entorno (García-Villacorta et al., 2003); sin embargo, los varillales en la Amazonia peruana se encuentran vulnerables y se están perdiendo o deteriorando por actividades antropogénicas, lo que ha causado la pérdida de algunas especies de aves endémicas para la región; de allí la importancia de evaluar este ecosistema para conocer la fauna aviar presente, poder determinar su singularidad y orientar la gestión y conservación de estas áreas (Álvarez, 2002), que son hábitat de numerosas especies de aves (Salazar et al., 2000; Rodríguez-Gamarra et al., 2003; Álvarez y Soini, 2007; Linna et al., 2008; Torres, 2011; Álvarez et al., 2013).
El mayor conocimiento de la biología de las aves del Varillal Alto Seco en la RNAM, incluyendo su morfometría, permitirán un mejor manejo y conservación de estas especies (Acosta, 2009). Así mismo, se resalta la importancia de realizar estudios a nivel de estratos, entre ellos el sotobosque, pues en los últimos 10 años se ha podido reportar más de dos docenas de especies nuevas para la ciencia, incluyendo cinco especies de aves y numerosas plantas e insectos (Álvarez, 2007). Por lo tanto, el presente trabajo tuvo como objetivo conocer la riqueza y características morfométricas de las aves de sotobosque en bosque de Varillal Alto Seco en la Reserva Nacional Allpahuayo Mishana.

\section{MATERIALes y Métodos}

El trabajo se realizó entre enero y mayo de 2016, en la formación boscosa denominada Varillal Alto Seco (VAS) de la Reserva 


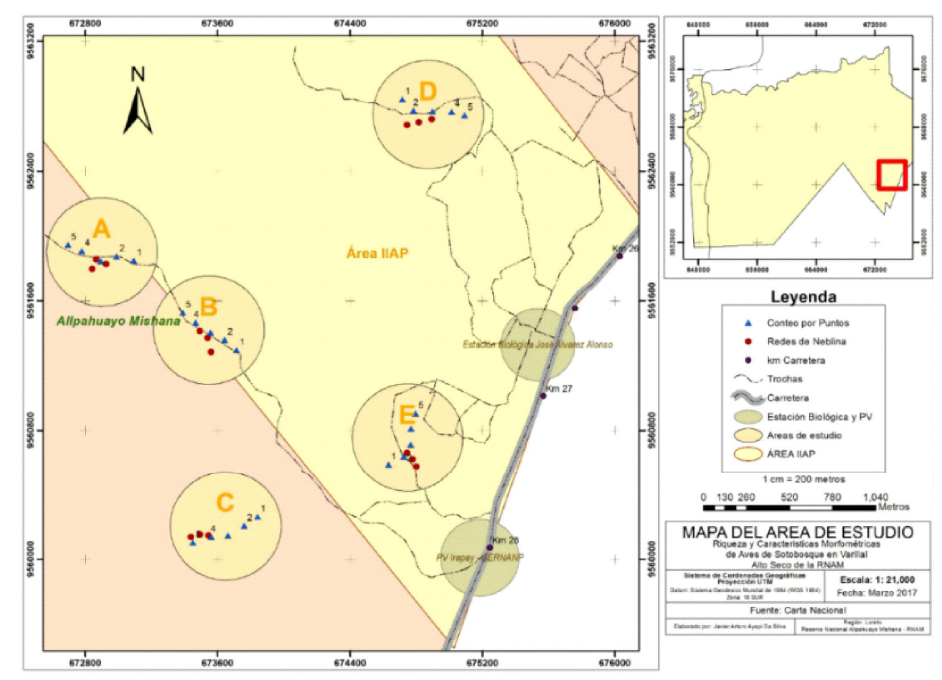

Figura 1. Mapa de ubicación del área de estudio y lugares de muestreo

Nacional Allpahuayo Mishana, a la altura del Puesto de Vigilancia «Irapay» (km 28 carretera Iquitos-Nauta, $9560009 \mathrm{~N}$ y $675184 \mathrm{E})$ y la Estación Biológica «José Álvarez Alonso (9561330N y 675534E) del Instituto de Investigaciones de la Amazonía Peruana (IIAP), km 26.7, al margen derecho de la carretera IquitosNauta. La RNAM se encuentra ubicada en el distrito de San Juan Bautista, provincia de Maynas, departamento de Loreto (Perú).

Se seleccionaron 5 VAS, identificados como A, B, C, D y E (Figura 1) donde se aplicaron las siguientes técnicas:

- Método de censo por puntos de conteo (Bibby et al., 1992). Se empleó para el registro de la avifauna observada y oída en sotobosque de varillal alto seco, en transecto de $1 \mathrm{~km}$ de largo con puntos distribuidos cada $100 \mathrm{~m}$, desde las 05:30 hasta las $12.00 \mathrm{y}$ desde las 15:00 hasta las 17:00 durante periodos de 10 minutos en cada punto de conteo, con un intervalo de 15 minutos para el traslado de un punto a otro. Así mismo, se emplearon binoculares Olympus de $5 \mathrm{x}$ $10^{\circ}$ para la observación de la avifauna.
- Método de captura de aves con redes de neblina (Bibby et al., 1992). Se emplearon 10 redes de neblina en cada muestreo $(12 \times 3 \mathrm{~m})$ con diámetro de malla estirada de 30 a $36 \mathrm{~mm}$. Las redes fueron colocadas a lo largo de transectos preexistentes y ocasionalmente se abrieron trochas de $15 \mathrm{~m}$, a una distancia de 40-60 m entre ellas, ubicando las redes a nivel de sotobosque (0-2.6 m). Las redes permanecieron abiertas entre las 05:30 y 12:00 y entre las 15:00 y 17:00 horas.

- Características morfométricas de las aves de sotobosque de VAS (Hilty y Brown, 1986). Se tomaron los datos morfométricos en las aves capturadas en las redes de neblina. Se determinó el peso (g) con una balanza digital CAMRY (precisión de $0.01 \mathrm{~g}$ ), así como la longitud del pico, longitud del culmen, longitud de la cabeza, longitud del ala, longitud de la cola y longitud del tarso empleando un vernier $(0.05 \mathrm{~mm}$ de precisión). Además, se hicieron registros fotográficos de las aves con una cámara digital Nikon de $16 \mathrm{MP}$. 


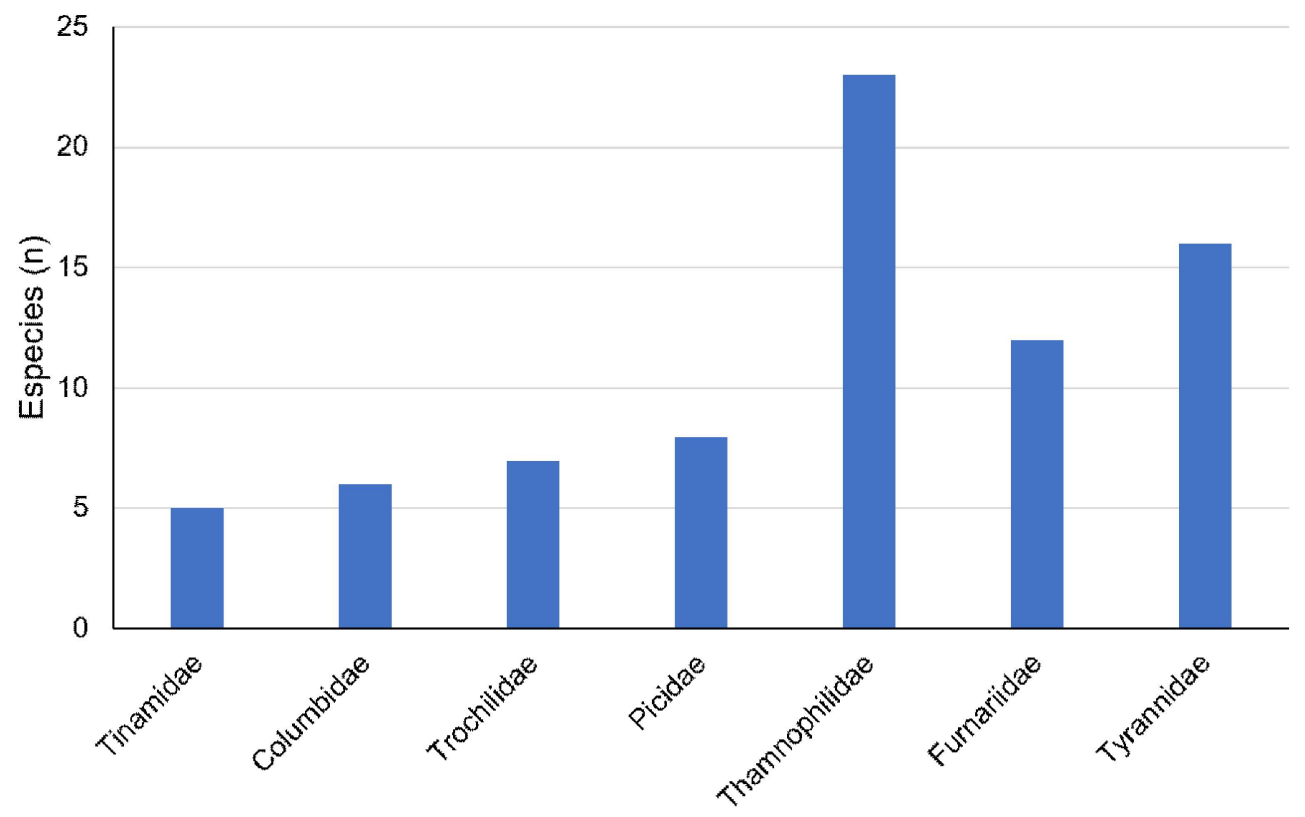

Familia

Figura 2. Principales familias y número de especies reportados en el Varillal Alto Seco de la Reserva Nacional Allpahuayo Mishana (2016)

Las aves observadas y capturadas fueron reconocidas según sus características morfológicas externas de acuerdo a Schulemberg et al. (2010) y la clasificación taxonómica según South American Classification Committee (2018). La riqueza de especies de aves se analizó mediante índices no paramétricos (Chao 1, Chao 2, Bootstrap y Jacknife 1) (Moreno, 2001), curva de acumulación de especies (curva de Clench) (Jiménez y Hortal, 2003), coeficiente de similaridad de Jaccard cualitativo, índices de diversidad de Margalef, ShannonWiener (Moreno, 2001), aplicando PAST v. 2.17. En el caso de las características morfométricas se utilizó la estadística descriptiva (valor mínimo y máximo, promedio y desviación estándar).

\section{Resultados}

\section{Riqueza de Especies de Aves}

La riqueza de especies de aves fue de 12 órdenes, 28 familias y 122 especies (considerando conteo por puntos y captura con redes). El Orden Passeriformes reportó 13 familias seguido de Caprimulgiformes, Coraciiformes y Galbuliformes con 2 familias respectivamente y el resto de las órdenes reportaron un menor número de familias. Así mismo, las familias Thamnophilidae, Furnariidae y Tyrannidae, todos pertenecientes al Orden Passeriformes resaltan en número de especies en el varillal alto seco (Figura 2); no obstante, en orden de importan- 
Cuadro 1. Índices de diversidad de especies de aves encontradas en cinco sectores (A-D) del Varillal Alto Seco de la Reserva Nacional Allpahuayo Mishana (2016)

\begin{tabular}{lccccc}
\hline & $\mathrm{A}$ & $\mathrm{B}$ & $\mathrm{C}$ & $\mathrm{D}$ & $\mathrm{E}$ \\
\hline Riqueza & 58 & 85 & 51 & 50 & 52 \\
Individuos & 121 & 143 & 88 & 116 & 86 \\
$\quad$ Índice de Shannon-Wiener (H')* & 3.87 & 4.30 & 3.83 & 3.69 & 3.76 \\
$\begin{array}{l}\text { Índice de diversidad de Simpson } \\
\text { (1-D)** }\end{array}$ & 0.97 & 0.98 & 0.97 & 0.96 & 0.97 \\
$\quad$ Índice de Margalef*** & 11.89 & 16.93 & 11.17 & 10.31 & 11.45 \\
\hline * Mide la diversidad de especies, considerando la uniformidad de estas \\
** Indica la relación existente entre el número de especies y la abundancia o número de Individuos \\
por especie \\
*** Estima la biodiversidad de una comunidad con base a la distribución numérica de los individuos \\
$\quad$ de las diferentes especies en función del número de individuos existentes en la muestra analizada
\end{tabular}

cia, también se destacan las familias Picidae, Trichilidae y Columbidae (Figura 2). Las familias que reportaron una sola especie fueron Cracidae, Odontophoridae, Conopophagidae, Vireonidae y Turdidae. La riqueza de especies por varillal muestreado (considerando los dos tipos de muestreo) varió entre 85 especies (varillal B) y 51 especies (varillal C) (Cuadro 1).

Considerando solo las especies capturadas con redes, se reporta una riqueza específica de 3 órdenes, 11 familias y 43 especies. Las órdenes reportadas fueron Galbuliformes, Piciformes y Passeriformes, siendo este último el que reportó ocho familias. La riqueza, según el muestreo con redes, varió entre 2 y 24 especies (Cuadro 1). En los cuadros 2, 3 y 4 se presenta la lista completa de órdenes, familias y especies registradas.

Los valores de los índices no paramétricos indican que el número de especies observadas estaban muy próximos a lo esperado. La riqueza específica de aves observadas (122 especies [sp]) más próxima a lo esperado fue CHAO 1 (130 sp), seguido de CHAO 2 (133 sp), Bootstrap (136 sp) y el más lejano fue Jackknife 1 (149 sp), lo cual indica que faltó realizar un mayor número de muestreos. La misma tendencia se observa aplicando la curva de Clench (función de acumulación) donde el número de especies esperadas fue 166 con respecto a las $122 \mathrm{ob}-$ servadas, pues con el número de muestreos realizados en cada varillal alto seco no se logra formar la asíntota; no obstante, el coeficiente de determinación $\left(\mathrm{R}^{2}\right)$ fue 0.999849074 , muy cercano a 1 , lo que indica un buen ajuste del modelo. Según los resultados de las especies observadas con respecto a las esperadas, solo se registró el $73.4 \%$ de la avifauna en el varillal alto seco, corroborado por el valor de la calidad del inventario de 0.020 (una pendiente menor de 0.1 indica que se ha logrado un inventario bastante completo y altamente fiable).

La dominancia de aves en el varillal alto seco es alta, según el índice de Simpson, con valores entre 0.96 y 0.98 , lo que indica una alta dominancia expresada por especies del Orden Passeriformes (72 especies) y una baja diversidad (rango entre 0 y 1 ). Así mismo, el índice de Margalef indica una alta diversidad en todos los varillales muestreados (valores mayores de 5 indican una alta diversidad), 
Cuadro 2. Listado completo de especies de aves registradas en el Varillal Alto Seco de la Reserva Nacional Allpahuayo Mishana (2016) - Parte I

\begin{tabular}{|c|c|c|c|c|}
\hline Orden & Familia & Especie & Nombre común & $\mathrm{n}$ \\
\hline Tinamiformes & Tinamidae & $\begin{array}{l}\text { Tinamus major } \\
\text { Tinamus guttatus } \\
\text { Crypturellus cinereus } \\
\text { Crypturellus soui } \\
\text { Crypturellus undulatus }\end{array}$ & $\begin{array}{l}\text { Perdiz grande } \\
\text { Perdiz de garganta blanca } \\
\text { Perdiz cinérea } \\
\text { Perdiz chica } \\
\text { Perdiz ondulada }\end{array}$ & $\begin{array}{l}3 \\
4 \\
2 \\
5 \\
4\end{array}$ \\
\hline Galliformes & $\begin{array}{l}\text { Cracidae } \\
\text { Odontophoridae }\end{array}$ & $\begin{array}{l}\text { Penelope jacquacu } \\
\text { Odontophorus gujanensis }\end{array}$ & $\begin{array}{l}\text { Pava de spix } \\
\text { Codorniz de cara roja }\end{array}$ & $\begin{array}{l}6 \\
14\end{array}$ \\
\hline Columbiformes & Columbidae & $\begin{array}{l}\text { Patagioenas plumbea } \\
\text { Patagioenas subvinacea } \\
\text { Leptotila verreauxi } \\
\text { Geotrigon montana } \\
\text { Columbina talpacoti } \\
\text { Claravis pretiosa }\end{array}$ & $\begin{array}{l}\text { Paloma plomiza } \\
\text { Paloma rojiza } \\
\text { Paloma de puntas blancas } \\
\text { Paloma perdiz rojiza } \\
\text { Tortolita rojiza } \\
\text { Tortolita azul }\end{array}$ & $\begin{array}{l}13 \\
2 \\
1 \\
1 \\
6 \\
4\end{array}$ \\
\hline Cuculiformes & Cuculidae & $\begin{array}{l}\text { Coccycua minuta } \\
\text { Piaya cayana }\end{array}$ & $\begin{array}{l}\text { Cuco menudo } \\
\text { Cuco ardilla }\end{array}$ & $\begin{array}{l}4 \\
4\end{array}$ \\
\hline Strigiformes & Strigidae & $\begin{array}{l}\text { Megascops choliba } \\
\text { Lophostrix cristata } \\
\text { Pulsatrix perspicillata } \\
\text { Ciccaba huhula }\end{array}$ & $\begin{array}{l}\text { Lechuza tropical } \\
\text { Búho penachudo } \\
\text { Búho de anteojos } \\
\text { Búho negro bandeado }\end{array}$ & $\begin{array}{l}2 \\
4 \\
5 \\
2\end{array}$ \\
\hline Caprimulgiformes & $\begin{array}{l}\text { Nyctibiidae } \\
\text { Caprimulgidae }\end{array}$ & $\begin{array}{l}\text { Nyctibius aethereus } \\
\text { Nyctibius griseus } \\
\text { Nyctidromus albicollis }\end{array}$ & $\begin{array}{l}\text { Nictibio de cola larga } \\
\text { Nictibio común } \\
\text { Chotacabras común }\end{array}$ & $\begin{array}{l}1 \\
2 \\
4\end{array}$ \\
\hline Apodiformes & Trochilidae & $\begin{array}{l}\text { Florisuga mellivora } \\
\text { Glaucis hirsutus } \\
\text { Threnetes leucurus } \\
\text { Phaethornis ruber } \\
\text { Phaethornis malaris } \\
\text { Heliodoxa aurescens } \\
\text { Thalurania furcata }\end{array}$ & $\begin{array}{l}\text { Colibrí de nuca blanca } \\
\text { Ermitaño de pecho canela } \\
\text { Ermitaño de cola pálida } \\
\text { Ermitaño rojizo } \\
\text { Ermitaño de pico grande } \\
\text { Brillante de pecho castaño } \\
\text { Ninfa de cola ahorquillada }\end{array}$ & $\begin{array}{l}7 \\
3 \\
1 \\
2 \\
7 \\
2 \\
3\end{array}$ \\
\hline Trogoniformes & Trogonidae & $\begin{array}{l}\text { Trogon viridis } \\
\text { Trogon ramonianus }\end{array}$ & $\begin{array}{l}\text { Trogón de dorso verde } \\
\text { Trogón amazónico }\end{array}$ & $\begin{array}{l}14 \\
6\end{array}$ \\
\hline Coraciiformes & $\begin{array}{l}\text { Alcedinidae } \\
\text { Momotidae }\end{array}$ & $\begin{array}{l}\text { Chloroceryle inda } \\
\text { Chloroceryle aenea } \\
\text { Electron platyrhynchum } \\
\text { Baryphthengus martii } \\
\text { Momotus momota }\end{array}$ & $\begin{array}{l}\text { Martín pescador verde y rufo } \\
\text { Martín pescador pigmeo } \\
\text { Relojero de pico ancho } \\
\text { Relojero rufo } \\
\text { Relojero amazónico }\end{array}$ & $\begin{array}{l}3 \\
1 \\
2 \\
2 \\
7\end{array}$ \\
\hline Galbuliformes & $\begin{array}{l}\text { Galbulidae } \\
\text { Bucconidae }\end{array}$ & $\begin{array}{l}\text { Jacamerops aureus } \\
\text { Galbula albirostris } \\
\text { Bucco capensis } \\
\text { Malacoptila fusca } \\
\text { Nonnula brunnea } \\
\text { Nonnula rubecula }\end{array}$ & $\begin{array}{l}\text { Jacamar grande } \\
\text { Jacamar de mejilla azul } \\
\text { Buco acollarado } \\
\text { Buco de pecho blanco } \\
\text { Monjita parda } \\
\text { Monjita de pecho rojizo }\end{array}$ & $\begin{array}{l}4 \\
6 \\
1 \\
3 \\
2 \\
1\end{array}$ \\
\hline Piciformes & Picidae & $\begin{array}{l}\text { Melanerpes cruentatus } \\
\text { Veniliornis affinis } \\
\text { Celeus elegans } \\
\text { Celeus grammicus } \\
\text { Celeus flavus } \\
\text { Dryocopus lineatus } \\
\text { Campephilus rubricollis } \\
\text { Campephilus melanoleucos }\end{array}$ & $\begin{array}{l}\text { Carpintero de penacho amarillo } \\
\text { Carpintero teñido de rojo } \\
\text { Carpintero castaño } \\
\text { Carpintero de pecho escamoso } \\
\text { Carpintero crema } \\
\text { Carpintero lineado } \\
\text { Carpintero de cuello rojo } \\
\text { Carpintero de cresta roja }\end{array}$ & $\begin{array}{l}13 \\
2 \\
10 \\
6 \\
2 \\
4 \\
6 \\
4\end{array}$ \\
\hline
\end{tabular}


Cuadro 3. Listado completo de especies de aves registradas en el Varillal Alto Seco de la Reserva Nacional Allpahuayo Mishana (2016) - Parte II

\begin{tabular}{|c|c|c|c|c|}
\hline Orden & Familia & Especie & Nombre común & $\mathrm{n}$ \\
\hline \multirow[t]{54}{*}{ Passeriformes } & \multirow[t]{23}{*}{ Thamnophilidae } & Thamnophilus murinus & Batará murino & 2 \\
\hline & & Thamnomanes caesius & Batará cinéreo & 3 \\
\hline & & Thamnomanes ardesiacus & Batará de garganta oscura & 1 \\
\hline & & Pygiptila stellaris & Batará de ala moteada & 1 \\
\hline & & Epinecrophylla haematonota & Hormiguerito de garganta punteada & 5 \\
\hline & & Myrmotherula longipennis & Hormiguerito de ala larga & 1 \\
\hline & & Myrmotherula axillaris & Hormiguerito de flanco blanco & 4 \\
\hline & & Hypocnemis peruviana & Hormiguero peruano & 3 \\
\hline & & Hypocnemis hypoxantha & Hormiguero de ceja amarilla & 1 \\
\hline & & Megastictus margaritatus & Batará perlado & 8 \\
\hline & & Isleria hauxwelli & Hormiguerito de garganta llana & 11 \\
\hline & & Dichrozona cincta & Hormiguerito bandeado & 2 \\
\hline & & Cercomacroides serva & Hormiguero negro & 2 \\
\hline & & Myrmoborus myotherinus & Hormiguero de cara negra & 5 \\
\hline & & Gymnopithys leucaspis & Hormiguero bicolor & 6 \\
\hline & & Sclateria naevia & Hormiguero plateado & 3 \\
\hline & & Schistocichla leucostigma & Hormiguero de ala moteada & 10 \\
\hline & & Myrmeciza fortis & Hormiguero tiznado & 2 \\
\hline & & Pithys albifrons & Hormiguero de plumón blanco & 15 \\
\hline & & Hylophylax naevius & Hormiguero de dorso moteado & 1 \\
\hline & & Rhegmatorhina melanosticta & Hormiguero de cresta canosa & 4 \\
\hline & & Willisornis poecilinotus & Hormiguero de dorso escamoso & 14 \\
\hline & & Phlegopsis nigromaculata & Ojo-pelado moteado de negro & 2 \\
\hline & \multirow[t]{2}{*}{ Formicariidae } & Formicarius analis & Gallito-hormiguero de cara negra & 3 \\
\hline & & Formicarius colma & Gallito-hormiguero de gorro rufo & 1 \\
\hline & \multirow[t]{2}{*}{ Furnariidae } & Sclerurus mexicanus & Tira-hoja de garganta anteada & 2 \\
\hline & & Sclerurus caudacutus & Tira-hoja de cola negra & 3 \\
\hline & \multirow[t]{10}{*}{ Furnariidae } & Glyphorynchus spirurus & Trepador pico de cuña & 27 \\
\hline & & Xiphorhynchus elegans & Trepador elegante & 3 \\
\hline & & Xiphorhynchus guttatus & Trepador de garganta anteada & 1 \\
\hline & & Dendrocincla merula & Trepador de barbilla blanca & 5 \\
\hline & & Dendrocincla fuliginosa & Trepador pardo & 1 \\
\hline & & Xenops minutus & Pico-lezna simple & 3 \\
\hline & & Dendrocolaptes certhia & Trepador barrado amazónico & 1 \\
\hline & & Anabacerthia ruficaudata & Limpia-follaje de cola rufa & 2 \\
\hline & & Certhiasomus stictolaemus & Trepador de garganta punteada & 3 \\
\hline & & Synallaxis rutilans & Cola espina rojizo & 1 \\
\hline & Conopophagidae & Conopophaga peruviana & Jejenero de garganta ceniza & 1 \\
\hline & \multirow[t]{16}{*}{ Tyrannidae } & Myiopagis gaimardii & Fío-fío de la selva & 2 \\
\hline & & Mionectes oleagineus & Mosquerito de vientre ocráceo & 8 \\
\hline & & Lophotriccus vitiosus & Tirano-pigmeo de doble banda & 6 \\
\hline & & Tolmomyias flaviventris & Pico-ancho de pecho amarillo & 2 \\
\hline & & Platyrinchus coronatus & Pico-chato de corona dorada & 2 \\
\hline & & Onychorhynchus coronatus & Mosquero real & 4 \\
\hline & & Pachyramphus marginatus & Cabezon de gorro negro & 1 \\
\hline & & Contopus virens & Pibí oriental & 3 \\
\hline & & Knipolegus poecilocercus & Viudita-negra amazónica & 4 \\
\hline & & Myiobius barbatus & Mosquerito de lomo azufrado & 3 \\
\hline & & Ramphotrigon ruficauda & Picoplano de cola rufa & 2 \\
\hline & & Myiozetetes similis & Mosquero social & 4 \\
\hline & & Myiozetetes granadensis & Mosquero de gorro gris & 2 \\
\hline & & Tyrannopsis sulphurea & Mosquero azufrado & 8 \\
\hline & & Terenotriccus erythrurus & Mosquerito de cola rojiza & 2 \\
\hline & & Attila spadiceus & Atila polimorfo & 5 \\
\hline
\end{tabular}


Cuadro 4. Listado completo de especies de aves registradas en el Varillal Alto Seco de la Reserva Nacional Allpahuayo Mishana (2016) - Parte III

\begin{tabular}{|c|c|c|c|c|}
\hline Orden & Familia & Especie & Nombre común & $\mathrm{n}$ \\
\hline \multirow[t]{17}{*}{ Passeriformes } & Cotingidae & Querula purpurata & Cuervo-frutero de garganta púrpura & $\begin{array}{l}4 \\
5\end{array}$ \\
\hline & \multirow[t]{6}{*}{ Pipridae } & Tyranneutes stolzmanni & Saltarín-tirano enano - chaufita & 5 \\
\hline & & Machaopterus regulus & Satarín rayado & 10 \\
\hline & & Lepidothrix coronata & Saltarín de corona azul & 21 \\
\hline & & Ceratopipra erythrocephala & Saltarín de cabeza dorada & 12 \\
\hline & & Dixiphia pipra & Saltarín de corona blanca & 10 \\
\hline & & Manacus manacus & Saltarín de barba blanca & 5 \\
\hline & \multirow[t]{3}{*}{ Tityridae } & Schiffornis turdina & Shifornis de ala parda & 4 \\
\hline & & Laniocera hypopyrra & Plañidero cinéreo & 3 \\
\hline & & Pachyramphus polychopterus & Cabezón de ala blanca & 1 \\
\hline & Vireonidae & Tunchiornis ochraceiceps & Verdillo de corona leonada & 1 \\
\hline & \multirow[t]{2}{*}{ Troglodytidae } & Campylorhynchus turdinus & Cucarachero zorzal & 6 \\
\hline & & Microcerculus marginatus & Cucarachero de pecho escamoso & 2 \\
\hline & Turdidae & Turdus albicollis & Zorzal de cuello blanco & 1 \\
\hline & \multirow{3}{*}{ Thraupidae } & Cissopis leverianus & Tangara urraca & 4 \\
\hline & & Ramphocelus carbo & Tangara de pico plateado & 20 \\
\hline & & Tachyphonus cristatus & Tangara cresta de fuego & 2 \\
\hline
\end{tabular}

Cuadro 5. Coeficiente de similaridad de Jaccard entre los puntos de muestreo en cinco varillales alto seco (A-D) de la Reserva Nacional Allpahuayo Mishana (2016)

\begin{tabular}{ccccc}
\hline A & B & C & D & E \\
\hline 1 & 0.31333 & 0.32773 & 0.5082 & 0.32824 \\
0 & 1 & 0.36885 & 0.26144 & 0.36567 \\
0 & 0 & 1 & 0.24194 & 0.22034 \\
0 & 0 & 0 & 1 & 0.41667 \\
0 & 0 & 0 & 0 & 1 \\
\hline
\end{tabular}

mientras que el índice de Shannon-Wiener indican una alta diversidad y una baja dominancia con valores que varían entre 3.69 y 4.30 (la diversidad es más alta cuanto más alto es su valor) (Cuadro 1). Por otro lado, se aprecia una baja similaridad en la composición de especies entre los varillales muestreados (Cuadro 5).

\section{Características Morfométricas}

Se colectaron 154 individuos de aves representados en 43 especies. Solo se observó un individuo por especie en 24 especies y más de dos individuos por especie en 19 especies (Cuadros 6 y 7). La mayoría de las aves colectadas fueron adultas (143 indivi- 
Cuadro 6. Datos morfométricos de especies de aves de sotobosque en Varillal Alto Seco de la Reserva Nacional Allpahuayo Mishana (2016). Medidas en milímetros y peso en gramos. Parte I

\begin{tabular}{|c|c|c|c|c|c|c|c|c|c|c|c|}
\hline 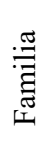 & Especie & $\mathrm{n}$ & Medida & $\begin{array}{l}\bar{\pi} \\
0 \\
0.0 \\
0 \\
0 \\
0\end{array}$ & 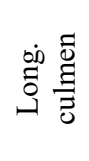 & $\begin{array}{l}\stackrel{0}{0} \\
\dot{0} \\
0.0 \\
\tilde{0} \\
.\end{array}$ & $\begin{array}{l}\frac{n}{\pi} \\
\frac{\pi}{\pi} \\
000 \\
\tilde{0} \\
1\end{array}$ & $\begin{array}{l}\frac{\pi}{0} \\
0 \\
0.0 \\
\tilde{0} \\
0\end{array}$ & 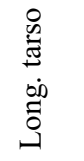 & 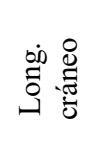 & Peso \\
\hline 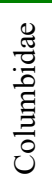 & $\begin{array}{l}\text { Geotrygon } \\
\text { montana }\end{array}$ & 3 & $\begin{array}{l}\text { Mín } \\
\text { Máx } \\
\text { Media } \\
\text { DE }\end{array}$ & $\begin{array}{c}184.5 \\
212 \\
198.8 \\
13.8\end{array}$ & $\begin{array}{c}7 \\
8.5 \\
7.8 \\
0.8\end{array}$ & $\begin{array}{c}18 \\
22 \\
20.7 \\
2.3\end{array}$ & $\begin{array}{c}118.3 \\
127 \\
123.5 \\
4.6\end{array}$ & $\begin{array}{c}66.8 \\
82 \\
75.2 \\
7.7\end{array}$ & $\begin{array}{c}25.4 \\
34.4 \\
29.3 \\
4.6\end{array}$ & $\begin{array}{c}23.1 \\
23.5 \\
23.2 \\
0.2\end{array}$ & $\begin{array}{c}28.5 \\
107.2 \\
77.0 \\
42.5\end{array}$ \\
\hline 苞 & $\begin{array}{l}\text { Celeus } \\
\text { elegans }\end{array}$ & 3 & $\begin{array}{l}\text { Mín } \\
\text { Máx } \\
\text { Media } \\
\text { DE }\end{array}$ & $\begin{array}{c}230 \\
263 \\
241.7 \\
18.5\end{array}$ & $\begin{array}{c}17.3 \\
22.5 \\
19.1 \\
3.0\end{array}$ & $\begin{array}{c}26 \\
34.9 \\
29.3 \\
4.9\end{array}$ & $\begin{array}{c}128 \\
153 \\
136.7 \\
14.2\end{array}$ & $\begin{array}{c}69 \\
94.9 \\
78.3 \\
14.4\end{array}$ & $\begin{array}{c}18.9 \\
24.8 \\
21.2 \\
3.2\end{array}$ & $\begin{array}{c}26.5 \\
30 \\
28.0 \\
1.8\end{array}$ & $\begin{array}{c}72 \\
153.3 \\
99.2 \\
46.8\end{array}$ \\
\hline \multirow{8}{*}{ 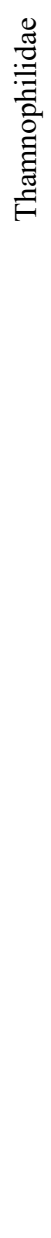 } & $\begin{array}{l}\text { Pithys } \\
\text { albifrons }\end{array}$ & 17 & $\begin{array}{l}\text { Mín } \\
\text { Máx } \\
\text { Media } \\
\text { DE }\end{array}$ & $\begin{array}{c}113 \\
160.3 \\
126 \\
11.4\end{array}$ & $\begin{array}{c}4.8 \\
16.3 \\
9.7 \\
3.6\end{array}$ & $\begin{array}{c}13.5 \\
20.7 \\
18.4 \\
2.3\end{array}$ & $\begin{array}{c}58.8 \\
70.5 \\
66.2 \\
3.5\end{array}$ & $\begin{array}{c}34 \\
50 \\
40.5 \\
3.9\end{array}$ & $\begin{array}{c}19.9 \\
27.5 \\
23.0 \\
1.8\end{array}$ & $\begin{array}{c}15 \\
37.7 \\
22.6 \\
7.2\end{array}$ & $\begin{array}{c}16.8 \\
26.9 \\
19.5 \\
2.2\end{array}$ \\
\hline & $\begin{array}{l}\text { Willisornis } \\
\text { poecilinotus }\end{array}$ & 9 & $\begin{array}{l}\text { Mín } \\
\text { Máx } \\
\text { Media } \\
\text { DE }\end{array}$ & $\begin{array}{c}120 \\
130 \\
126.8 \\
3.4\end{array}$ & $\begin{array}{c}7 \\
12.2 \\
10.2 \\
1.8\end{array}$ & $\begin{array}{c}15 \\
24.9 \\
18.7 \\
3.1\end{array}$ & $\begin{array}{c}58.6 \\
65 \\
62.2 \\
2.6\end{array}$ & $\begin{array}{c}38 \\
44 \\
41.5 \\
2.4\end{array}$ & $\begin{array}{c}21 \\
26.8 \\
23.8 \\
1.8\end{array}$ & $\begin{array}{c}15.1 \\
23 \\
18.8 \\
2.3\end{array}$ & $\begin{array}{c}16.4 \\
19.3 \\
17.6 \\
1.0\end{array}$ \\
\hline & $\begin{array}{l}\text { Megastictus } \\
\text { margaritatus }\end{array}$ & 4 & $\begin{array}{l}\text { Mín } \\
\text { Máx } \\
\text { Media } \\
\text { DE }\end{array}$ & $\begin{array}{c}126 \\
138 \\
131.6 \\
5.4\end{array}$ & $\begin{array}{c}11 \\
12.5 \\
11.7 \\
0.6\end{array}$ & $\begin{array}{c}22 \\
22.9 \\
22.3 \\
0.4\end{array}$ & $\begin{array}{c}62.7 \\
73 \\
69.6 \\
4.7\end{array}$ & $\begin{array}{c}47 \\
50.5 \\
48.6 \\
1.5\end{array}$ & $\begin{array}{c}19.2 \\
20.9 \\
20.2 \\
0.8\end{array}$ & $\begin{array}{c}20 \\
23 \\
21.2 \\
1.3\end{array}$ & $\begin{array}{c}20.3 \\
23.0 \\
21.3 \\
1.3\end{array}$ \\
\hline & $\begin{array}{l}\text { Myrmoborus } \\
\text { myotherinus }\end{array}$ & 2 & $\begin{array}{l}\text { Mín } \\
\text { Máx } \\
\text { Media } \\
\text { DE }\end{array}$ & $\begin{array}{c}117.0 \\
117.0 \\
117.0 \\
0.0\end{array}$ & $\begin{array}{c}10.8 \\
12.3 \\
11.6 \\
1.1\end{array}$ & $\begin{array}{c}20.0 \\
21.2 \\
20.6 \\
0.8\end{array}$ & $\begin{array}{c}56.9 \\
61.2 \\
59.1 \\
3.0\end{array}$ & $\begin{array}{c}32.5 \\
38.2 \\
35.4 \\
4.0\end{array}$ & $\begin{array}{c}24.5 \\
25.1 \\
24.8 \\
0.4\end{array}$ & $\begin{array}{c}19.9 \\
22.1 \\
21.0 \\
1.6\end{array}$ & $\begin{array}{c}19.0 \\
32.6 \\
25.8 \\
9.6\end{array}$ \\
\hline & $\begin{array}{l}\text { Myrmotherula } \\
\text { axilaris }\end{array}$ & 4 & $\begin{array}{l}\text { Mín } \\
\text { Máx } \\
\text { Media } \\
\text { DE }\end{array}$ & $\begin{array}{c}93.0 \\
110.0 \\
101.4 \\
7.0\end{array}$ & $\begin{array}{l}3.0 \\
8.7 \\
7.0 \\
2.7\end{array}$ & $\begin{array}{c}10.4 \\
17.1 \\
14.7 \\
3.0\end{array}$ & $\begin{array}{c}46.6 \\
52.6 \\
50.8 \\
2.8\end{array}$ & $\begin{array}{c}34.3 \\
37.0 \\
35.6 \\
1.2\end{array}$ & $\begin{array}{c}16.0 \\
16.8 \\
16.4 \\
0.4\end{array}$ & $\begin{array}{c}14.8 \\
26.9 \\
18.4 \\
5.7\end{array}$ & $\begin{array}{l}7.7 \\
9.6 \\
8.8 \\
0.8\end{array}$ \\
\hline & $\begin{array}{l}\text { Epinecrophylla } \\
\text { haematonota }\end{array}$ & 3 & $\begin{array}{l}\text { Mín } \\
\text { Máx } \\
\text { Media } \\
\text { DE }\end{array}$ & $\begin{array}{c}103.0 \\
111.0 \\
108.0 \\
4.4\end{array}$ & $\begin{array}{c}9.6 \\
10.3 \\
9.9 \\
0.4\end{array}$ & $\begin{array}{c}13.3 \\
19.0 \\
17.1 \\
3.3\end{array}$ & $\begin{array}{c}48.0 \\
51.0 \\
50.0 \\
1.7\end{array}$ & $\begin{array}{c}35.0 \\
35.5 \\
35.3 \\
0.3\end{array}$ & $\begin{array}{c}15.5 \\
16.5 \\
16.0 \\
0.5\end{array}$ & $\begin{array}{c}13.3 \\
16.0 \\
15.1 \\
1.5\end{array}$ & $\begin{array}{c}8.9 \\
10.0 \\
9.7 \\
0.6\end{array}$ \\
\hline & $\begin{array}{l}\text { Islerya } \\
\text { hauswelli }\end{array}$ & 6 & $\begin{array}{l}\text { Mín } \\
\text { Máx } \\
\text { Media } \\
\text { DE }\end{array}$ & $\begin{array}{c}81.4 \\
105.0 \\
89.9 \\
8.1\end{array}$ & $\begin{array}{c}7.1 \\
11.0 \\
9.0 \\
1.3\end{array}$ & $\begin{array}{c}16.0 \\
22.0 \\
18.4 \\
2.2\end{array}$ & $\begin{array}{c}47.2 \\
54.0 \\
49.9 \\
2.4\end{array}$ & $\begin{array}{c}18.8 \\
23.5 \\
21.6 \\
1.6\end{array}$ & $\begin{array}{c}15.4 \\
20.1 \\
17.9 \\
2.2\end{array}$ & $\begin{array}{c}17.3 \\
18.9 \\
17.9 \\
0.5\end{array}$ & $\begin{array}{c}10.9 \\
12.3 \\
11.4 \\
0.5\end{array}$ \\
\hline & $\begin{array}{l}\text { Schistocichla } \\
\text { leucostigma }\end{array}$ & 3 & $\begin{array}{l}\text { Mín } \\
\text { Máx } \\
\text { Media } \\
\text { DE }\end{array}$ & $\begin{array}{c}134.0 \\
160.0 \\
143.3 \\
14.5\end{array}$ & $\begin{array}{c}11.0 \\
18.6 \\
13.9 \\
4.1\end{array}$ & $\begin{array}{c}21.0 \\
24.0 \\
22.7 \\
1.5\end{array}$ & $\begin{array}{l}59.0 \\
84.0 \\
69.3 \\
13.1\end{array}$ & $\begin{array}{c}52.0 \\
64.4 \\
58.2 \\
6.2\end{array}$ & $\begin{array}{c}25.0 \\
26.6 \\
25.9 \\
0.8\end{array}$ & $\begin{array}{l}- \\
- \\
- \\
-\end{array}$ & $\begin{array}{c}25.7 \\
35.3 \\
29.6 \\
5.1\end{array}$ \\
\hline \multirow[t]{2}{*}{ 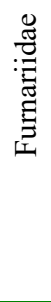 } & $\begin{array}{l}\text { Glyphorynchus } \\
\text { spirurus }\end{array}$ & 26 & $\begin{array}{l}\text { Mín } \\
\text { Máx } \\
\text { Media } \\
\text { DE }\end{array}$ & $\begin{array}{c}124.4 \\
151.0 \\
139.7 \\
7.0\end{array}$ & $\begin{array}{c}3.0 \\
15.5 \\
9.8 \\
2.9\end{array}$ & $\begin{array}{c}10.0 \\
25.0 \\
16.1 \\
3.2\end{array}$ & $\begin{array}{c}56.0 \\
72.0 \\
66.7 \\
3.7\end{array}$ & $\begin{array}{c}57.3 \\
75.0 \\
67.1 \\
4.4\end{array}$ & $\begin{array}{c}13.0 \\
28.0 \\
17.4 \\
2.8\end{array}$ & $\begin{array}{c}14.5 \\
26.1 \\
17.2 \\
2.3\end{array}$ & $\begin{array}{c}12.2 \\
15.4 \\
13.8 \\
0.9\end{array}$ \\
\hline & $\begin{array}{l}\text { Xiphorhynchus } \\
\text { elegans }\end{array}$ & 4 & $\begin{array}{l}\text { Mín } \\
\text { Máx } \\
\text { Media } \\
\text { DE }\end{array}$ & $\begin{array}{c}177.0 \\
280.0 \\
235.0 \\
43.4\end{array}$ & $\begin{array}{c}14.2 \\
29.5 \\
23.8 \\
6.6\end{array}$ & $\begin{array}{c}23.9 \\
38.7 \\
33.7 \\
7.0\end{array}$ & $\begin{array}{c}79.9 \\
151.0 \\
115.5 \\
30.1\end{array}$ & $\begin{array}{c}81.1 \\
126.0 \\
101.3 \\
19.2\end{array}$ & $\begin{array}{c}20.0 \\
27.5 \\
23.5 \\
3.1\end{array}$ & $\begin{array}{c}21.1 \\
25.6 \\
23.3 \\
2.0\end{array}$ & $\begin{array}{l}20.9 \\
62.3 \\
44.9 \\
18.9\end{array}$ \\
\hline
\end{tabular}


Cuadro 7. Datos morfométricos de especies de aves de sotobosque en Varillal Alto Seco de la Reserva Nacional Allpahuayo Mishana (2016). Medidas en milímetros y peso en gramos. Part II

\begin{tabular}{|c|c|c|c|c|c|c|c|c|c|c|c|}
\hline 营 & Especie & $\mathrm{n}$ & Medida & 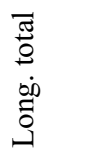 & 过 & $\begin{array}{l}\stackrel{0}{0} \\
\stackrel{0}{0} \\
0.0 \\
\tilde{0} \\
.\end{array}$ & $\begin{array}{l}\frac{0}{\sigma} \\
\frac{\pi}{\pi} \\
00 \\
0 \\
0 \\
.0\end{array}$ & $\begin{array}{l}\frac{\pi}{0} \\
0 \\
0.0 \\
0 \\
0 \\
0\end{array}$ & 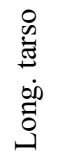 & 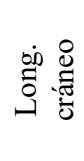 & Peso \\
\hline \multirow{8}{*}{ 苞 } & Mionectes & 3 & Mín & 106.0 & 8.0 & 14.2 & 52.4 & 40.6 & 13.8 & 15.2 & 9.2 \\
\hline & oleagineus & & Máx & 108.0 & 11.9 & 19.5 & 58.7 & 48.0 & 16.0 & 16.3 & 10.8 \\
\hline & & & Media & 106.9 & 9.4 & 17.0 & 56.4 & 45.0 & 14.7 & 16.0 & 10.2 \\
\hline & & & $\mathrm{DE}$ & 1.0 & 2.2 & 2.7 & 3.5 & 3.9 & 1.2 & 0.6 & 0.9 \\
\hline & Attila & 2 & Mín & 160.0 & 4.0 & 14.3 & 79.0 & 69.0 & 24.2 & 23.4 & 31.5 \\
\hline & spadiceus & & Máx & 178.0 & 14.2 & 27.9 & 80.2 & 71.2 & 24.2 & 26.2 & 31.5 \\
\hline & & & Media & 169.0 & 9.1 & 21.1 & 79.6 & 70.1 & 24.2 & 24.8 & 31.5 \\
\hline & & & $\mathrm{DE}$ & 12.7 & 7.2 & 9.6 & 0.9 & 1.6 & 0.0 & 2.0 & 0.0 \\
\hline \multirow{16}{*}{ : } & Manacus & 5 & Mín & 77.0 & 3.2 & 10.0 & 46.0 & 15.0 & 13.5 & 17.5 & 10.1 \\
\hline & manacus & & Máx & 109.0 & 7.6 & 14.3 & 59.0 & 32.5 & 22.0 & 18.8 & 17.9 \\
\hline & & & Media & 97.1 & 5.6 & 12.5 & 53.0 & 22.2 & 17.5 & 18.0 & 14.3 \\
\hline & & & $\mathrm{DE}$ & 12.5 & 2.1 & 2.3 & 4.7 & 8.3 & 4.0 & 0.7 & 3.2 \\
\hline & Pipra & 3 & Mín & 81.4 & 8.8 & 16.0 & 47.2 & 21.4 & 15.4 & - & - \\
\hline & erythrocephala & & Máx & 90.5 & 9.4 & 18.4 & 54.0 & 21.8 & 16.5 & - & - \\
\hline & & & Media & 85.6 & 9.1 & 17.2 & 50.6 & 21.6 & 15.9 & - & - \\
\hline & & & $\mathrm{DE}$ & 4.6 & 0.3 & 1.2 & 3.4 & 0.2 & 0.6 & - & - \\
\hline & Dixiphia & 10 & Mín & 83.0 & 2.0 & 7.8 & 52.1 & 19.9 & 12.1 & 16.5 & 10.8 \\
\hline & pipra & & Máx & 120.0 & 10.2 & 18.0 & 66.0 & 29.0 & 21.0 & 20.5 & 13.5 \\
\hline & & & Media & 100.1 & 7.3 & 14.2 & 62.3 & 25.5 & 15.0 & 17.9 & 12.1 \\
\hline & & & $\mathrm{DE}$ & 9.3 & 2.2 & 2.6 & 3.9 & 3.1 & 2.4 & 1.1 & 1.0 \\
\hline & Lepidotrhix & 21 & Mín & 76.0 & 4.0 & 5.5 & 49.9 & 21.5 & 11.5 & 14.1 & 7.8 \\
\hline & coronata & & Máx & 100.0 & 8.6 & 15.0 & 59.1 & 32.5 & 19.0 & 27.3 & 11.4 \\
\hline & & & Media & 88.7 & 6.3 & 11.7 & 55.2 & 26.4 & 15.0 & 17.2 & 9.1 \\
\hline & & & $\mathrm{DE}$ & 6.6 & 1.2 & 2.0 & 2.2 & 3.0 & 1.9 & 3.9 & 0.9 \\
\hline \multirow{4}{*}{ 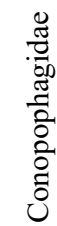 } & Conopophaga & 3 & Mín & 104.0 & 8.0 & 14.0 & 59.5 & 23.5 & 23.0 & 18.9 & 22.8 \\
\hline & peruviana & & Máx & 113.5 & 9.5 & 18.0 & 63.0 & 26.8 & 29.8 & 22.5 & 24.0 \\
\hline & & & Media & 109.8 & 8.8 & 16.5 & 61.8 & 25.1 & 25.6 & 20.1 & 23.3 \\
\hline & & & $\mathrm{DE}$ & 5.1 & 0.8 & 2.2 & 2.0 & 1.7 & 3.7 & 2.1 & 0.6 \\
\hline
\end{tabular}

duos) y el resto fueron juveniles como los casos de Platyrinchus coronatus (1), Rupornis magnirostris (1), Dixiphia pipra (1) y Lepidothrix coronata (8). Por otra parte, 45 individuos fueron machos, 51 individuos fueron hembras y en 58 individuos no se pudo determinar el sexo, especialmente en las especies que carecían de dimorfismo sexual basado en el patrón de coloración. Con relación al número de individuos capturados por especies, destacan Glyphorynchus spirurus (26 individuos) Lepidothrix coronata (21), Pithys albifrons (17) y Dixiphia pipra (10).

\section{Discusión}

Los datos reportados en el presente trabajo difieren con lo reportado para varillales de la parte superior del río Tigre (Perú) y de la RNAM con respecto a la especie Herpsi- 
lochmus gentryi (Álvarez y Whitney, 2003), que no fue observado ni capturado en el presente trabajo debido a que es un ave que frecuenta el dosel, ni tampoco fue reportado Crypturellus duidae (Álvarez y Whitney, 2003), donde probablemente el área de distribución de esta especie sería en los varillales cercanos al río Nanay, área distante de la zona de influencia donde se realizó el presente trabajo. Algo similar ocurrió con la especie Zimmerius villarejoi, que no fue reportada en el presente trabajo, aunque podría ser encontrada dentro de la cuenca del rio Nanay y en partes de la RNAM, ya que es considerada como un ave poco común (Whitney y Álvarez, 2002).

Con respecto a los varillales alto seco evaluados en la RNAM, los resultados son muy superiores con respecto a los resultados de diversidad y abundancia de aves de sotobosque de varillal alto seco del Centro de Investigaciones Allpahuayo (CIA) (Torres, 2011; Álvarez et al., 2013; Fong et al., 2014), pero coinciden con dos de las familias más representativas (Thamnophilidae y Furnariidae), mientras que difiere con las familias Pipridae y Tyrannidae. Así mismo son semejantes con las especies más frecuentes y abundantes: Glyphorynchus spirurus, Pithys albifrons, Lepidothrix coronata y Pipra pipra (Dixiphia pipra) y con dos especies categorizadas como especialistas de arena blanca: Megastictus margaritatus y Pipra pipra (Dixiphia pipra) de las cuatro reportadas para los varillales alto seco del CIA (Megastictus margaritatus, Myrmeciza castanea, Sclerurus rufigularis y Pipra pipra) (Fong et al., 2014).

Con relación a las medidas biométricas, la bibliografía especializada solo reporta datos de longitud total (Whitney y Alvarez, 2002; McMullan y Donegan, 2014), dificultando el análisis comparativo, aunque Hilty y Brown (1986) mencionan datos adicionales. La longitud total (que representa el tamaño de un ave desde la punta del pico hasta la punta de la cola) de las especies reportadas en el presente trabajo están dentro del rango reporta- do para individuos colectados en Colombia (Hilty y Brown, 1986; McMullan y Donegan, 2014), con ligeras variaciones debido a que dichas medidas biométricas fueron tomadas de especímenes de museo. Cabe indicar que la longitud de un ave se encuentra influenciada tanto por la longitud del pico como por la longitud de la cola (Schulemberg et al., 2010).

\section{Conclusiones}

- La riqueza de especies en el Varillal Alto Seco de la Reserva Nacional Allpahuayo Mishana está compuesto por 122 especies con dominancia del orden Passeriformes (13 familias y 72 especies) y con una baja similaridad entre los varillales estudiados debido a un elevado número de especies que solo cuentan con una o dos individuos respectivamente en el inventario (singletons, doubletons) y únicas.

- Los datos morfométricos indican que los individuos de las especies están dentro de los rangos reportados para individuos de otras localidades (Colombia) y dentro de la Amazonia peruana.

- Los datos del presente estudio ayudarán a discriminar las diferencias interespecíficas de las especies similares y tener más precisión en el reconocimiento taxonómico.

\section{Agradecimientos}

Los autores agradecen a la Jefatura de la Reserva Nacional Allpahuayo Mishana y la Estación Biológica José Álvarez Alonso IIAP por las facilidades brindadas para la realización del estudio.

\section{Literatura Citada}

1. Acosta DA. 2009. Bioecología de Dendrobates reticulatus en el varillal alto seco de la Reserva Nacional 
Allpahuayo-Mishana, Iquitos. Tesis Doctoral. Lima: Univ. Nacional Federico Villareal. $201 \mathrm{p}$.

2. Alvarez J. 2002. Characteristic avifauna of white-sand forest in northern Peruvian Amazonia. MSc Thesis. Baton Rouge, LA, USA: Lousiana State Uni-versity. 156 p.

3. Alvarez J 2007. Reserva Nacional Allpahuayo-Mishana: una joya natural al lado de Iquitos. Iquitos, Perú: IIAP. 80 p.

4. Alvarez, J, Whitney M. 2003. New distributional record of birds from whitesand forests of the northern Peruvian amazon, with implications for biogeography of northern South America. The Condor 105: 552-566. doi: 10.1650/7159

5. Álvarez J, Soini P. 2007. Importancia de la Zona Reservada AllpahuayoMishana para la conservación de la biodiversidad de la amazonia peruana, Loreto, Perú. Iquitos, Perú: IIAP. 16 p.

6. Alvarez J, Metz M, Fine PVA. 2013. Habitat specialization by birds in western Amazonian white-sand forests. Biotropica 45: 365-372. doi: 10.1111/btp.12020

7. Bibby CJ, Burges N, Hill D. 1992. Bird census techniques. London: Academic Press. $125 \mathrm{p}$.

8. Fong E, Herrera R, Acosta A. 2014. Diversidad de aves de sotobosque de varillal alto seco en el Centro de Investigaciones Allpahuayo - IIAP Loreto. Conoc Amaz 5: 101-113.

9. García Villacorta R, Ahuite-Reátegui M, Olortegui-Zumaeta M. 2003. Clasificación de bosques sobre arena blanca en la Zona Reservada AllpahuayoMishana. Folia Amazónica 14: 17-33. doi: 10.24841/fa.v14i1.151

10. Hilty SL, Brown W. 1986. A guide to the birds of Colombia. USA: Pinceton University Press. 836 p.

11. Jiménez A, Hortal J. 2003. Las curvas de evaluación silvestre y la necesidad de evaluar la calidad de los inventarios biológicos. Rev Ibérica Aracnología 8: 151-161.
12. Linna A, Nuotio K, Rinne J, Salo M,; Saaksjarvi I, Bendayan D, Rojas J, Rodríguez A, Torres M. 2008. Estudio piloto: factibilidad del uso de redes de neblina para documentación de la avifauna en la Reserva Nacional Allpahuayo-Mishana. Finlandia: Universidad de Turku. $16 \mathrm{p}$.

13. McMullan M, Donegan T. 2014. Field guide to the birds of Colombia. Colombia: Fundación ProAves. 396 p.

14. Moreno C. 2001. Métodos para medir la biodiversidad. Acta Zool Mex 85: 195-195.

15. Rodríguez-Gamarra JK, Ruokolainen K, Soini P, Salo J. 2003. La diversidad biológica de la Zona Reservada Allpahuayo Mishana, Loreto, Perú. Folia Amazónica 14: 101-103. 10.24841/ fa.v14i1.156

16. Salazar E, Mattos J, Díaz J, Ferreyra F, Piana R, Balta K. 2000. Composición de especies de aves en hábitats de la zona reservada Allpahuayo-Mishana y colinas de la formación nauta. Folia Amazónica 14: 125-132.

17. Schulemberg T, Stotz D, Lane D, O'Neill J, Parker T. 2010. Birds of Peru. USA: Princeton University Press. $660 \mathrm{p}$.

18. South American Classification Committee. 2018. A classification of the bird species of South America. [Internet]. Disponible en: http:// www.museum.1su.edu/ Remsen/ SACCBaseline.htm

19. Torres M. 2011. Patrones de muda de la avifauna en la Reserva Nacional Allpahuayo Mishana. Loreto, Perú. Tesis. Perú: Univ. Nacional de la Amazonía Peruana. 57 p.

20. Whitney BM, Alvarez J. 2002. New species of gnatcatcher from white sand forests of northern Amazonian Perú with revision of the Polioptila guianensis complex. Wilson Bull 117: 113-127. doi: 10.1676/04-064 